\title{
Recurso humano altamente educado en Guatemala Escenarios y retos ${ }^{1}$
}

\author{
Kleinsy Yudrani Bonilla Landaverry \\ Facultad de Postgrados en Estudios Internacionales Pan-Pacific GSP \\ Universidad Kyung Hee, República de Corea del Sur \\ kleinsy@gmail.com \\ Kwak Jae-Sung \\ Facultad de Postgrados en Estudios Internacionales Pan-Pacific GSP \\ Universidad Kyung Hee, República de Corea del Sur
}

Fecha de recepción: 29/08/2014

Fecha de aceptación: 02/10/2014

\begin{abstract}
Resumen
Este artículo pretende ofrecer una valoración crítica de la disponibilidad, formación y aprovechamiento de los recursos humanos nacionales altamente educados (RRHHAE) en Guatemala. Para el propósito de este artículo, se entiende a los RRHHAE en términos de logro educativo y el desarrollo de competencias entre la población; en última instancia, enmarcado en la educación superior. El enfoque se ha colocado en los recursos humanos graduados de programas académicos de cuarto nivel (maestría, doctorado). Los autores inician proveyendo una visión general del contexto del país; secciones subsiguientes reflexionan sobre el perfil de desarrollo de Guatemala y los desafíos enfrentados por su sistema de educación superior que obstaculizan su capacidad para producir los necesarios RRHHAE para el desarrollo de la nación. Los hallazgos proporcionan evidencia de la escasez de RRHHAE en Guatemala, así como la existencia de condiciones que hacen que sea poco probable que se produzcan internamente. Además plantean los diferentes elementos del escenario de trabajo que limitan el mejor aprovechamiento de los RRHHAE guatemaltecos. Este estudio busca contribuir a una mejor comprensión de los RRHHAE de Guatemala, la importancia de la cooperación internacional para hacer frente a las deficiencias en el ámbito nacional, y sugiere varios temas para futuras investigaciones.
\end{abstract}

Palabras clave: Gestión de recursos humanos, desarrollo de la capacidad humana, cooperación para el desarrollo, estudios de postgrado en Guatemala, recurso humano altamente educado, educación superior en Guatemala.

\begin{abstract}
This article attempts to provide a critical valuation of the availability, formation and best use of national highly educated human resources (HEHHRR) in Guatemala. HEHHRR, for the purpose of this article, is understood in terms of educational attainment and skills development among the population; ultimately framed in higher education. Focus has been placed on individuals graduated from fourth-level academic programs (master, doctoral degrees). The authors start by providing an overview of the country context; subsequent sections reflect on the development profile of Guatemala and the challenges faced by its domestic higher education system which hinder its ability to comply with its role in the production of the needed HEHHRR for the development of the nation. Findings provide evidence of the scarcity of HEHHRR in Guatemala as well as the existence of conditions that make it unlikely to produce them domestically. In addition, different elements of the work scenario limit the best use of the Guatemalan HEHHRR. Therefore, this study strives to provide a better insight into the HEHHRR of Guatemala, the importance of international cooperation to address shortcomings at the domestic level, and suggests various topics for future research.
\end{abstract}

Key words: Human resource management, human capacity development, development cooperation, postgraduate education in Guatemala, highly educated human resources in Guatemala, higher education in Guatemala

\footnotetext{
${ }^{1}$ En base a su versión en inglés por el Asian Journal of Latin American Studies (2014), Vol. 27 No. 3: 17-43
} 


\section{Introducción}

Guatemala muestra algunos de los indicadores más bajos en relación a la formación de recursos humanos en la región latinoamericana -un área en sí misma rezagada de otras regiones emergentes, como el Nordeste, Sudeste y Centro de Asia-. La producción de magísteres y doctores guatemaltecos en el sistema de educación superior nacional ha demostrado ser ineficiente e insuficiente. La investigación científica y las actividades orientadas a la academia no parecen ser consideradas prioridad en la vida pública y en el debate social de este país centroamericano (Hernández y Blass, 2013). La falta de políticas públicas relacionadas con la gestión RRHHAE, un desestructurado y débil sistema de educación terciaria, la falta de relación entre los sectores privado, público y académico, así como la inexistencia de planificación junto a una visión de corto plazo, son algunos de los factores que explican en parte las enormes adversidades que hay que superar en este campo.

Asumiendo que el sistema de educación superior de Guatemala es responsable de la formación de las próximas generaciones de empresarios, líderes políticos, profesionales y la fuerza de trabajo crítica que liderará el camino hacia el desarrollo de Guatemala, es fundamental hacer frente a los varios desafíos mencionados; esto ya ha sido reconocido por expertos (Aldana, 2014; Rodríguez, 2014; Arenas, 2012), académicos (Sacayón, 2012; Tobar, 2011; Godínez y Tobar, 2005), funcionarios de gobierno y líderes políticos (Parrilla, 2013; Rubio, 2013; Fuentes, 2013) y autoridades de educación superior (Medina, 2014; Cabarrus, 2013; Ponce, 2007; Gálvez, 2006).

Los diferentes sectores de la sociedad guatemalteca han sido testigos de la progresiva decadencia de la educación superior. Periodistas y medios de comunicación han llamado en varias ocasiones la atención del país a estas cuestiones (González, 2013; León 2013; Iteriano, 2012; Hurtado, 2011). Esto es consistente con los desafortunados indicadores que Guatemala registra constantemente en diferentes informes relacionados con la formación de recursos humanos en las universidades nacionales (Brunner, 2011; Retana y Esquivel, 2006; Steier y Yammal, 2001). En la problemática a abordar, algunos elementos destacados son: la débil gobernanza y un marco jurídico obsoleto, insuficiente cobertura, la desigualdad, la baja calidad de la educación, la participación de la política partidista en el sector académico y la ineficiencia.

Una fuerza de trabajo altamente educada y hábil es vital para alcanzar objetivos de desarrollo más amplios. Es por ello que este estudio intenta responder a tres preguntas concretas: 1. ¿Cuál es la disponibilidad de recursos humanos altamente educados en Guatemala? 2. ¿En qué medida el sistema de educación superior nacional de Guatemala es capaz de producir los recursos humanos altamente educados que necesita?, y 3 . ¿Cuál es el sector del contexto de trabajo que permite el mejor aprovechamiento de los RRHHAE para el desarrollo de Guatemala?

El objetivo de este artículo es examinar el estado actual de la educación superior y la formación de RRHHAE en Guatemala, que aparentemente ha sido incapaz de cumplir con su responsabilidad. La situación desalentadora del sistema de educación superior en Guatemala, junto con el objetivo de desarrollar la capacidad humana ha motivado a varios miembros de la comunidad de donantes a cooperar con Guatemala desde 1980.

Recientemente más países desarrollados se han sumado a este esfuerzo. En este artículo se aplica una metodología de investigación cualitativa para permitir el análisis de los desafíos que enfrenta Guatemala para fortalecer el talento humano, esto como un requisito para tener éxito en el nuevo paradigma de una economía del conocimiento, ello mismo perfila la esperanza proporcionada por el apoyo de los donantes para hacer frente a esos desafíos. 


\section{Revisión de literatura}

\section{a. Perfil de desarrollo de Guatemala}

Guatemala se encuentra ubicada en América Central, limita con México al norte y al oeste, el Océano Pacífico al sudoeste, Belice y el Mar Caribe al noreste, y Honduras y El Salvador al sureste. El país tiene una extensión territorial de $108,889 \mathrm{~km}^{2}$, de los cuales el $98,4 \%$ es suelo y el $1.6 \%$ restante es agua. La capacidad de uso de la tierra se inclina $39.4 \%$ al uso de la agricultura y el $34,1 \%$ para la cobertura forestal (BCIE, 2010). El territorio está dividido en 22 provincias conocidas como departamentos y 333 municipios. La creencia religiosa predominante se divide en cristianismo, entre las religiones católica $(60 \%)$, protestantes $(30 \%)$ y la minoría (10\%) (Oficina de Democracia, Derechos Humanos y Trabajo, 2009). El Banco Mundial (2013) clasifica las economías según su ingreso. Hay países de bajos ingresos con un ingreso nacional bruto per cápita de $\$ 1,025$ o menos; de renta media baja, \$1,026- \$4,035; de ingresos medios altos, $\$ 4$ 4,036- 12.475; y altos ingresos, 12,476 dólares. Guatemala es considerado en la categoría de renta media baja, con un ingreso calculado para 2012 de $\$ 4,700$.

En cuanto al contexto social, Guatemala presenta algunos de los peores indicadores en materia de educación y salud en América Central (una región con los indicadores más bajos en América Latina y el Caribe). La tasa de analfabetismo es la más alta con un $25,2 \%$ de la población mayor de 15 años de edad. La educación es también una evidencia del atraso de la educación en el país, con la educación primaria todavía no se alcanza una cobertura del $100 \%$ al quedarse corto con un $95,1 \%$; la tasa neta de matriculación en la educación secundaria no es más que el $39,9 \%$. Una alta tasa de deserción en la escuela secundaria deja matrícula de educación secundaria en sólo el 17,7\% (WB, 2008, 2013).

Tabla 1 Guatemala, Indicadores Socioeconómicos

\begin{tabular}{|c|c|c|c|c|c|c|c|c|c|}
\hline Indicadores Socioeconómicos & 2000 & 2001 & 2002 & 2003 & 2004 & 2005 & 2006 & $2010^{*}$ & $2012^{*}$ \\
\hline PBI crecimiento (anual \%) & 3.6 & 2.3 & 2.2 & 2.1 & 2.7 & 3.2 & 4.5 & 3.8 & 3.1 \\
\hline $\begin{array}{c}\text { INB per cápita, PPP } \\
\text { (internacional actual \$) }\end{array}$ & 4.310 & 4.440 & 4.460 & 4.540 & 4.680 & 4.860 & 5.120 & 4.620 & 4.760 \\
\hline $\begin{array}{c}\text { Inflación, PBI deflactor (anual } \\
\text { \%) }\end{array}$ & 6.8 & 7.6 & 8.0 & 6.3 & 7.0 & 7.8 & 6.3 & 5.1 & 5.5 \\
\hline Gasto Militar (\% de PIB) & 0.8 & 0.9 & 0.7 & 0.7 & 0.4 & 0.3 & 0.4 & 0.4 & 0.4 \\
\hline $\begin{array}{c}\text { Expectativa de Vida al nacer } \\
\text { total (años) }\end{array}$ & 67.9 & - & 68.9 & - & - & 69.7 & 69.9 & 74 & 75 \\
\hline Población en millones & 11.2 & 11.5 & 11.8 & 12.1 & 12.4 & 12.7 & 13.0 & 14.7 & - \\
\hline $\begin{array}{c}\text { Crecimiento de la población } \\
\text { (anual \%) }\end{array}$ & 2.4 & 2.4 & 2.5 & 2.5 & 2.5 & 2.5 & 2.5 & 2.02 & 1.95 \\
\hline $\begin{array}{c}\text { Total tasa de fertilidad } \\
\text { (nacimientos por mujer) }\end{array}$ & 4.8 & - & 4.6 & - & - & 4.3 & 4.2 & 4.0 & 3.9 \\
\hline $\begin{array}{c}\text { VIH prevalencia (\% población } \\
\text { entre 15-49) }\end{array}$ & - & - & - & 0.9 & - & 0.9 & - & 0.7 & 0.8 \\
\hline $\begin{array}{c}\text { Escuela Primaria indicador } \\
\text { de conclusión (\% del grupo } \\
\text { relevante) }\end{array}$ & 55.7 & 60.3 & 64.1 & 65.2 & 69.8 & 73.7 & 76.5 & 84 & 86 \\
\hline $\begin{array}{c}\text { Tasa de niñas con relación a } \\
\text { niños en escuela primaria } \\
\text { (\%) }\end{array}$ & 88.9 & 89.6 & 90.7 & 90.6 & 91.1 & 91.6 & 92.3 & 95 & - \\
\hline $\begin{array}{c}\text { Remesas de trabajadores y } \\
\text { compensación recibida (\% } \\
\text { PIB) }\end{array}$ & 3.1 & 3.0 & 6.9 & 8.6 & 9.5 & 9.6 & 10.3 & 10.23 & 9.6 \\
\hline
\end{tabular}

Fuente: World Bank, 2008, * 2013 
Con relación al desarrollo humano el índice (IDH) de Guatemala para el año 2012 era 0.581 en la categoría de desarrollo humano medio posicionando al país en la casilla 133 de los 187 países y territorios considerados. Entre 1980 y 2012, el valor de IDH de Guatemala aumentó de 0,432 a 0,581, un aumento del 34 por ciento o aumento medio anual de alrededor del 0,9 por ciento (PNUD, 2013). Para una perspectiva general de los indicadores socioeconómicos de Guatemala, la tabla 1 incluye información relevante para la última década. Algunos indicadores han registrado una mejora constante, como la esperanza de vida al nacer, la tasa de conclusión de la educación primaria y la estabilidad microeconómica. Por el contrario, el indicador de desigualdad es uno de los más altos del mundo; en Guatemala el decil más pobre de la población recibe sólo el 1,0\% del ingreso nacional, mientras que el decil más rico recibe el 47,4\% (BCIE, 2010).

La economía de Guatemala continúa estructurada en el modelo agroexportador de desarrollo manteniendo una alta dependencia de los productos agrícolas y las exportaciones tradicionales de bajo valor agregado. Desde su independencia, la agroexportación como modelo económico ofrece pocos incentivos a la innovación basada en la tecnología local. Por siglos se ha privilegiado la importación de conocimiento en lugar de su producción nacional. Desde su independencia en 1821 la mayor parte de la economía se ha basado en la agricultura. Guatemala es el mayor exportador de cardamomo, el quinto exportador de azúcar y el séptimo exportador de café en todo el mundo (FUNDESA, 2012). La agricultura es complementada por el turismo, convirtiéndose en la segunda fuente de divisas después de las remesas.

Casi 1.637.119 inmigrantes económicos guatemaltecos se han movilizado sobre todo a Estados Unidos $(97,4 \%)$ en busca de oportunidades de trabajo, lo que significa el $11 \%$ de la población de Guatemala (UNICEF, 2010). Cinco ramas explican el $70 \%$ de la producción total del país (FUNDESA, 2012). La Figura 1 refleja una economía en la que el capital humano y la innovación juegan un papel limitado. En comparación con otras economías de la región que están promoviendo y mejorando su matriz productiva hacia el conocimiento y los servicios y el alto valor agregado, tales como Chile, Ecuador y Colombia, el caso de Guatemala sigue siendo subdesarrollado. El PIB de Guatemala está dominado por el sector privado, que genera el $85 \%$. La agricultura todavía representa el $75 \%$ de las exportaciones.

Figura 1 Guatemala, Actividad Económica (\%)

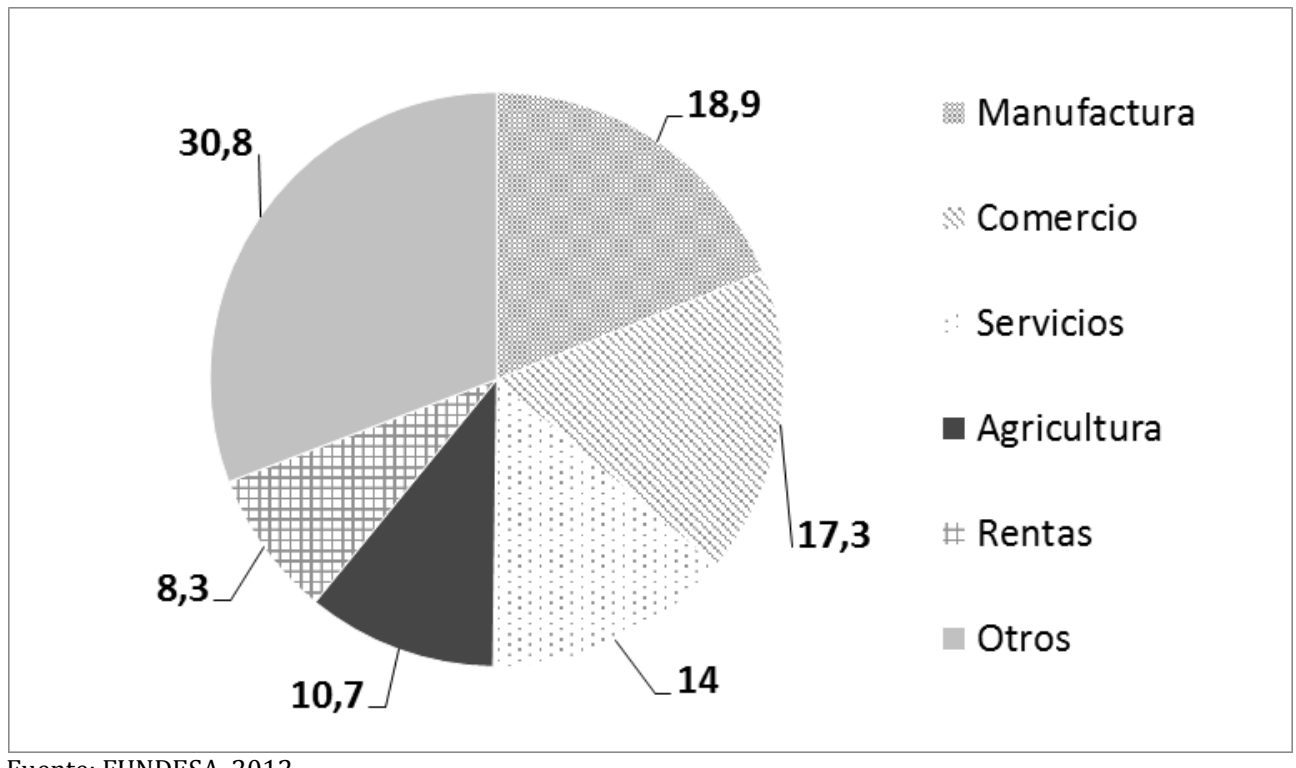

Fuente: FUNDESA, 2012 
En términos de competitividad Guatemala se enfrenta a importantes desafíos cuando se trata de innovación, la fortaleza institucional, la educación superior, desarrollo de habilidades y la formación de su recurso humano. Algunos indicadores elaborados para evaluar aspectos de la competitividad (Foro Económico Mundial, 2012) consideran que una economía es impulsada por la eficiencia desarrollando procesos de producción más eficaces y aumentando la calidad del producto, porque los salarios han aumentado y no pueden competir con precios, este es el caso de Guatemala. En este punto, la competitividad es impulsada cada vez más por la educación superior y la formación, los mercados de bienes eficientes, el buen funcionamiento de las estructuras de trabajo, y la capacidad de aprovechar los beneficios de las tecnologías existentes. Esto no está pasando en Guatemala. En la parte baja de la clasificación, en el lugar 830, Guatemala aumentó un solo lugar en 2010 De acuerdo con el Foro Económico Mundial (2012: 34), uno de los factores más problemáticos para hacer negocios en Guatemala es la insuficientemente educada fuerza laboral. Este aspecto afecta negativamente a las condiciones que ofrece el país para hacer negocios.

".. El país cuenta con algunas fortalezas relativas de competitividad en términos de regulaciones laborales flexibles para contratar y despedir personal (54aㅡ y la determinación de los salarios (43 $\left.3^{\mathrm{a}}\right)$, el desarrollo del mercado financiero eficiente $\left(41^{\mathrm{a}}\right)$, y la intensidad de la competencia local (46 $\stackrel{\text { a }}{ }$ ). Sin embargo, su competitividad se ve obstaculizada por la debilidad del público institucional $\left(130^{a}\right.$ ) y obstaculizado por los altos costos de la delincuencia y la violencia (144) y la baja confianza de la comunidad empresarial en los políticos (122 aㅡ). Nivel muy bajo de capacidad de innovación de Guatemala es el resultado de un sistema de baja calidad de la educación (130), escaso uso de las TIC (99 a $)$, y las inversiones de innovación relacionados-D bajo investigación y (90 a). La débil calidad de sus infraestructuras de transporte (93a) también afecta negativamente a su competitividad nacional." (Foro Económico Mundial, 2012: 34)

Cada año, 140.000 jóvenes guatemaltecos entran en el mercado laboral, entre ellos sólo
25.000 acceden al empleo formal, formación y la seguridad social. Por los últimos 10 años, sólo se han creado 200.000 nuevos puestos de trabajo, que confirman la prevalencia del empleo informal de 2,9 millones de dólares en 1.989 a 5.100 .000 de habitantes en 2011.

\section{b. Retos de la Educación Superior en Guatemala}

No se encontraron fuentes académicas que aborden la producción de RRHHAE en Guatemala. La mayoría de los artículos escritos son considerados de prensa y artículos de opinión (Medina, 2014; González, 2013, Arenas, 2012; Sacayón, 2012; Hurtado, 2011). Con un énfasis general en la enseñanza en lugar de las actividades de investigación, las universidades guatemaltecas también han limitado los intentos serios para estudiar de forma sistemática y con rigor científico los resultados de las instituciones de educación superior en Guatemala (Aldana, 2014; Hernández y Blas, 2013; Tobar, 2011).

Godínez y Tobar (2005) proporcionan un diagnóstico general del sistema de educación de postgrado para la educación superior pública en Guatemala. Esto es particularmente significativo ya que en Guatemala sólo hay una universidad pública que inscribe el $42 \%$ de la población total de estudiantes universitarios, mientras que las 14 universidades privadas restantes cubren el 58\% (Tobar, 2011: 20). En su estudio Godínez y Tobar (2005) concluyen que tanto la Universidad de San Carlos de Guatemala como las universidades privadas en el país ofrecen programas académicos de postgrado insuficientes que al mismo tiempo están excesivamente concentrados en las ciencias sociales. Estos programas, según los autores mencionados, no son sólo insuficientes, sino también ineficientes. La relación entre la matrícula y graduación observada durante el período de estudio (1992-2004) reflejan un índice sostenido de 0.1306 lo que significa que de cada 100 estudiantes de posgrado inscritos en San Carlos de la Universidad de 
Guatemala sólo 13 completaron con éxito sus programas de obtención de la respectiva especialización, maestría o doctorado. Godínez y Tobar (2005) también reconocen los retos que enfrentan quienes se gradúan de programas académicos superiores avanzados al afrontar la búsqueda de empleo en Guatemala. De acuerdo con su estudio las estructuras económicas, productivas e industriales en el país ofrecen pocas oportunidades de empleo. Esta situación presenta el dilema del huevo y la gallina al momento de decidir qué es lo primero: contar con una cantidad considerable de RRHHAE guatemalteca que atraiga la inversión productiva, que al mismo tiempo dará empleo a ellos o que la industria debe evolucionar primero con el fin de crear los incentivos que motiven en los guatemaltecos la participación en programas de postgrado.

Calderón (2005) aborda los problemas de repetición y deserción en la educación superior de Guatemala. Tanto en la universidad pública como en las privadas esta problemática es fundamental en términos de ineficacia e ineficiencia de la educación terciaria, los estudiantes universitarios no terminan sus carreras o toman demasiado tiempo y recursos en hacerlo. A nivel de postgrado al nivel de estado incompleto es más crítica. Calderón (2005: 18) concluye que los resultados fueron "desalentadores para la educación superior de Guatemala, el 96,5\% de la población carece de títulos de educación terciaria y los que están inscritos tienen que superar diferentes obstáculos con el fin de completar sus programas. Más del $75 \%$ de los estudiantes universitarios en Guatemala se ven afectados por el abandono o deserción." No sólo el acceso a la educación superior es un problema, sino también lo es la finalización con éxito de los programas.

Las organizaciones internacionales y regionales de educación (UNESCO, 2012; Moreno y Ruiz, 2009; UNESCO / IESALC, 2006) han puesto de relieve las reformas que la educación superior necesita con urgencia en América Latina, América Central y Guatemala. En particular, el caso de
Guatemala se señaló como uno de los países que muestra rezago. Varios indicadores de la situación de la educación superior en Guatemala ni siquiera pueden ser analizados debido a la falta de información y registros sistemáticos (Brunner, 2009). Cuando existe información, los indicadores presentan también cómo el sistema de educación superior en Guatemala sufre de baja cobertura, la desigualdad, la exclusión y una marcada desconexión entre la academia y las necesidades de la sociedad (Knut, 2009; UNESCO / IESALC, 2006, Rama, 2006).

Tobar (2011) presenta un estudio exhaustivo titulado "La Educación Superior en Guatemala en la Primera Década del Siglo XXI". Sus datos y análisis concluyen que todo el sistema de educación superior en Guatemala no es tal cosa. En lugar de un sistema de educación superior en Guatemala la oferta de educación terciaria es proporcionada por un número insuficiente de universidades desvinculadas unas de otras sin evaluación adecuada o aseguramiento de la calidad. Tobar (2011) reconoce que es cierto que el número de universidades autorizadas para operar en Guatemala ha crecido recientemente, sin embargo los datos de cobertura no son esperanzadores ya que los indicadores son mucho más bajos que el promedio de América Latina. La educación superior aún no ha cumplido su papel para convertirse en el motor para resolver los grandes retos nacionales [para el desarrollo de Guatemala] desde su creación hasta hoy su impacto es insignificante" (Tobar, 2011: 73). La educación terciaria en Guatemala aún responde al siglo pasado sin la incorporación de nuevas tecnologías y mejores prácticas. Adicionalmente, la cantidad limitada de la inversión pública en la educación superior, un desequilibrio significativo entre las regiones urbanas y rurales y su acceso a la educación superior, la desigualdad y el débil gobierno universitario dificulta la formación en educación terciaria entre los guatemaltecos; además, la producción de RRHHAE para el desarrollo del país se ve comprometida.

Sacayón (2012) también analiza los grandes desafíos que enfrenta la educación superior 
en Guatemala sosteniendo que Guatemala "adolece de déficits en el acceso a la educación superior, una parcial y selectiva oferta académica perpetúa el modelo económico actual del país".

Este modelo económico no responde a las exigencias de la moderna economía del conocimiento, sino al modelo agroexportador con poco valor agregado a la producción guatemalteca. Teniendo en cuenta la población de entre 15 y 29 años sólo el $6 \%$ tiene acceso a la educación superior. De cada 100 guatemaltecos sólo 12 alcanzan al menos un año de educación superior. Esto se agrava cuando se considera la población desagregada por etnia, urbano-rural, género y campos académicos.

Según Sacayón (2012), la falta de información, la inexistencia de registros fiables en las universidades, la ausencia de una institución pública a cargo de la coordinación y evaluación de la oferta de educación superior en Guatemala se encuentran entre las cuestiones urgentes que deben abordarse, estos asuntos son urgentes en lo que respecta principalmente al acceso y cobertura sin siquiera considerar la calidad de la educación superior, el nivel de formación de los profesores universitarios y la eficiencia del sistema.

\section{Metodología}

La metodología aplicada en esta investigación fue de tipo cualitativa, con énfasis en las experiencias de los participantes. La postura que prevalece es un análisis interpretativo basado en el esclarecimiento de la realidad en las opiniones de los participantes. Los datos fueron recogidos a través de tres métodos: a. Entrevistas personales, b. Encuesta en línea, y c. Investigación documental.

\section{a. Entrevistas}

La investigación de campo se llevó a cabo en Guatemala, de diciembre de 2012 a febrero de 2013, en un período de 10 semanas que permitió la recolección de datos de primera mano, lo que incluye la aplicación de entrevistas personales semi-estructuradas y abiertas. Un segundo período de investigación de campo se llevó a cabo del 1 al 14 de febrero de 2014. La tabla 2 contiene los detalles de las personas entrevistadas. Por medio de este método de investigación se recogió información de las principales partes interesadas y que participan en el sistema de educación superior de Guatemala.

Los participantes fueron invitados a participar en el estudio sobre la base de su experiencia de trabajo, ya sea en el sector público, la academia, las instituciones y organizaciones relacionadas con la estructura, la regulación, las políticas o la prestación de la educación superior en Guatemala.

Las entrevistas se llevaron a cabo en los lugares sugeridos por los entrevistados de forma personal, con una duración promedio de 75 minutos. Las preguntas incluidas en los instrumentos de investigación se relacionaron con consultas sobre el diagnóstico del sistema de educación superior en Guatemala y su progreso en la formación de RRHHAE para el desarrollo del país. ${ }^{2}$.

\footnotetext{
${ }^{2}$ Para una copia de los cuestionarios pueden contactar a los autores.
} 
Tabla 2 Lista de Entrevistados- Actores clave en el Sistema de Educación Superior $\mathbf{n = 1 2}$

\begin{tabular}{|c|c|c|c|}
\hline Organización & Nombre & Cargo & $\begin{array}{l}\text { Fecha de } \\
\text { Entrevista }\end{array}$ \\
\hline $\begin{array}{l}\text { Universidad de San Carlos de } \\
\text { Guatemala -USAC- }\end{array}$ & $\begin{array}{l}\text { María del Rosario } \\
\text { Godínez }\end{array}$ & Sistema de Estudios de Postgrados USAC & 2013.02.04 \\
\hline $\begin{array}{l}\text { Universidad de San Carlos de } \\
\text { Guatemala -USAC- }\end{array}$ & Cesar Morataya & $\begin{array}{l}\text { Coordinador del Departamento de Becas y } \\
\text { entrenamiento con Cooperación } \\
\text { Internacional }\end{array}$ & 2013.02.06 \\
\hline $\begin{array}{l}\text { Universidad de San Carlos de } \\
\text { Guatemala -USAC- }\end{array}$ & $\begin{array}{l}\text { María Teresa Molina } \\
\text { Santos }\end{array}$ & $\begin{array}{l}\text { Coordinadora de Convenios y } \\
\text { Negociaciones Cooperación International }\end{array}$ & 2013.02.06 \\
\hline $\begin{array}{l}\text { Universidad de San Carlos de } \\
\text { Guatemala -USAC- }\end{array}$ & Nohemí Luz Navas & $\begin{array}{l}\text { Jefe del Departamento de Evaluación y } \\
\text { Promoción Docente }\end{array}$ & 2013.02.08 \\
\hline $\begin{array}{l}\text { Universidad de San Carlos de } \\
\text { Guatemala -USAC- }\end{array}$ & Efraín Medina & $\begin{array}{l}\text { Ex Rector de la Universidad de San Carlos } \\
\text { de Guatemala }\end{array}$ & 2014.14.11 \\
\hline $\begin{array}{l}\text { Universidad de San Carlos de } \\
\text { Guatemala -USAC- }\end{array}$ & Mario Rodríguez & Instituto de Problemas Nacionales & 2014.02 .13 \\
\hline $\begin{array}{l}\text { Universidad de San Carlos de } \\
\text { Guatemala -USAC- }\end{array}$ & José Gramajo & Director de Evaluación Académica & 2014.02 .13 \\
\hline $\begin{array}{l}\text { Consejo Superior de } \\
\text { Universidades de Centroamérica } \\
\text {-CSUCA- }\end{array}$ & $\begin{array}{l}\text { Francisco Alarcón } \\
\text { Alba }\end{array}$ & Sub-Secretario General & 2013.02.26 \\
\hline $\begin{array}{l}\text { Universidad Rafael Landívar } \\
\text {-URL- }\end{array}$ & $\begin{array}{l}\text { Carlos Rafael } \\
\text { Cabarrus }\end{array}$ & Vice-rector de Investigación & 2013.02.16 \\
\hline Ministerio de Educación & Carlos Aldana & Ex Viceministro de Educación & 2014.02 .13 \\
\hline $\begin{array}{l}\text { Instituto para el Desarrollo de la } \\
\text { Educación Superior en } \\
\text { Guatemala - INDESGUA- }\end{array}$ & Luis Edgar Arenas & Director General & 2013.01.05 \\
\hline $\begin{array}{l}\text { Secretaría Nacional de Ciencia y } \\
\text { Tecnología SENACYT }\end{array}$ & $\begin{array}{l}\text { Miriam Patricia } \\
\text { Rubio }\end{array}$ & Secretaria Nacional SENACYT & 2013.10.22 \\
\hline
\end{tabular}

\section{b. Encuesta en línea}

Los suscriptores del Instituto para el Desarrollo de la Educación Superior en Guatemala -INDESGUA- fueron invitados a rellenar una encuesta en línea titulada: "Aprovechamiento de los recursos humanos guatemaltecos altamente educados (magísteres y doctores) para el desarrollo del país". De los más de 10.000 suscriptores de INDESGUA, cerca de 1,000 están preparando o han presentado solicitudes para estudiar programas de postgrado. El período de la encuesta abarcó desde 22 hasta 28 de febrero de 2014. Durante el período en que la encuesta en línea estaba disponible 518 encuestados la completaron.

La encuesta incluyó cuatro preguntas relacionadas con tres temas principales: 1. Disponibilidad de recursos humanos guatemaltecos con educación superior 2 . Sector de empleo que mejor aprovecha los recursos humanos guatemaltecos con educación superior para el desarrollo del país, y 3. Calificación del nivel de prioridad de ocho campos académicos / de conocimiento. Las preguntas 1, 2 y 3 fueron presentados como opción múltiple y la pregunta 4 pidió a los encuestados asignar un nivel de prioridad a cada uno de los ámbitos académicos / de conocimientos siendo la máxima prioridad 1 y 8 la mínima. Complementariamente, se prestó una opción para exponer los motivos de la elección de la pregunta 2 para recoger datos cualitativos sobre los mejores usos y desafíos para el aprovechamiento de los recursos humanos guatemaltecos con educación superior para el desarrollo del país.

\section{c. Investigación documental}

La investigación documental involucró la obtención y análisis de datos que ya existían, los planes del Gobierno de Guatemala y los informes emitidos por las instituciones 
públicas en Guatemala, así como universidades y otras organizaciones.

\section{Resultados y discusión}

Tres temas principales fueron identificados a través del análisis de datos. Los entrevistados respondieron a la encuesta en línea y presentaron sus percepciones con respecto a:

a. Disponibilidad de RRHHAE en Guatemala y el papel del sistema de educación superior nacional en la formación de RRHHAE b. Sector de empleo que mejor aprovecha los RRHHAE para el desarrollo de Guatemala c. Prioridad de campos académicos/ conocimiento para el desarrollo de Guatemala

La investigación documental proporcionó datos complementarios. Los tres temas se discuten en esta sección para establecer sus implicaciones en el contexto de la guatemalteca RRHHAE.

a. Disponibilidad de RRHHAE y el papel del Sistema de Educación Superior doméstica en la Formación de RRHHAE

Los recursos humanos guatemaltecos altamente educados se han considerado tradicionalmente como parte de la élite. Durante la época colonial entre 1,523 y 1,821 sólo los descendientes directos de los conquistadores españoles tenían acceso a la educación superior, sólo pocos ciudadanos podrían considerarse académicos 0 estudiosos. Más tarde, ya que Guatemala se independizó, se mantuvo una exclusión sistemática de las mujeres, los indígenas y los pobres. Fue hasta la Revolución de 1944 que la educación superior se hizo más accesible para los menos privilegiados. Incluso hoy en día, Guatemala refleja en la educación de su gente la desigualdad que prevalece en su estructura social y económica.

En general, la fragmentación de las instituciones involucradas en la educación superior y la inexistencia de un marco legal integral $y$ actualizado que regulen la educación superior en Guatemala han afectado la cobertura, calidad y resultados de la educación terciaria en el país. $\mathrm{El}$ instrumento jurídico más importante de Guatemala: la Constitución Política de la República asignó en 1,985 a la única universidad pública en el país la regulación general de la educación superior. Se trata de la Universidad de San de Guatemala -USAC- fundada el 31 de enero de 1,676. La USAC es una de las más antiguas instituciones de educación superior en América Latina. Con el mismo instrumento legal se creó el Consejo de Educación Superior Privada -CEPS- como el órgano encargado de evaluar las iniciativas para crear nuevas universidades privadas. Por último, la Constitución creó en 1,985 las 11 Asociaciones profesionales (Colegios Profesionales) que regulan el ejercicio profesional de sus miembros. Hay 13 universidades privadas autorizadas para operar en Guatemala. La tabla 3 a continuación muestra las instituciones de educación superior nacionales existentes en el país.

Estas tres estructuras: USAC, CEPS, las 13 universidades privadas actualmente en funcionamiento (véase la tabla 3 ) y Colegios Profesionales son las únicas instituciones relacionadas con la educación superior en Guatemala. Coordinación con instituciones externas es inexistente, en su mayoría funcionan de forma independiente, sin control y supervisión real. 
Tabla 3 Instituciones de Educación Superior en Guatemala

\begin{tabular}{|l|l|}
\hline Universidad & Fecha de Fundación \\
\hline San Carlos de Guatemala & $31 / 01 / 1976$ \\
\hline Rafael Landivar & $18 / 10 / 1961$ \\
\hline Del Valle de Guatemala & $29 / 01 / 1966$ \\
\hline Mariano Galvez & $01 / 06 / 1966$ \\
\hline Francisco Marroquin & $12 / 08 / 1971$ \\
\hline Rural de Guatemala & $28 / 03 / 1995$ \\
\hline Del Istmo & $19 / 09 / 1997$ \\
\hline Panamericana & $10 / 02 / 1998$ \\
\hline Mesoamericana & $01 / 10 / 1999$ \\
\hline Galileo & $31 / 10 / 2000$ \\
\hline San Pablo de Guatemala & $27 / 06 / 2006$ \\
\hline Inter Naciones & $06 / 08 / 2009$ \\
\hline De Occidente & $01 / 01 / 2011$ \\
\hline Da Vinci & $13 / 11 / 2012$ \\
\hline
\end{tabular}

Fuente: Consejo para la Educación Privada Superior http://www.ceps.edu.gt/ceps

El Ministerio de Educación de Guatemala no tiene competencia en el tratamiento de la educación superior, ya que sólo regula y organiza educación preprimaria, primaria y secundaria. Steier y Yammal (2001), sostienen que "el marco jurídico del sistema de educación superior de Guatemala está desactualizado y fue fuertemente afectada por los acontecimientos históricos, en particular la de 36 años de conflicto interno". Esto es consistente con Chamarbawala (2010) que en su estudio analiza los impactos negativos de la guerra civil sobre las instituciones de formación de capital humano, los trabajadores profesionales y los académicos de Guatemala. La falta de rendición de cuentas en el uso de los recursos públicos por parte de la USAC y la inexistencia de controles de calidad y las normas también dificultan la planificación a largo plazo, la eficacia y la eficiencia.

Otro problema acuciante es la falta de regulación sobre la estructura, las instituciones, el control de calidad y el incremento de la cobertura. Es uno de los pocos países de América Latina sin una Ley general de Educación Superior, tal como existe en la mayoría de las naciones vecinas. Como se ha indicado antes, el Ministerio de Educación y de cualquier otra institución del sector público no tienen participación en la educación superior. La competencia para crear y desarrollar políticas públicas en la educación superior se ha concedido a la única universidad nacional: USAC.

Otras cuestiones que afectan a la educación superior de la Guatemala son similares a las sufridas por la mayoría de los países en desarrollo como un marco jurídico obsoleto, falta de liderazgo y el poder de los organismos reguladores y la coordinación inexistente entre las 13 universidades privadas autorizadas que operan en el país.

La educación superior en Guatemala es una asignatura pendiente que requiere atención urgente por parte de sus autoridades. Los desafíos en diferentes áreas como la cobertura y el acceso, la oferta académica, la calidad de la educación, la consistencia de la educación universitaria con las necesidades de la sociedad han sido señaladas por académicos, políticos, periodistas y especialistas (Hurtado, 2011; Piril, 2011; Moreno Brid y Ruiz-Nápoles, 2009; López, 2004). Uno de los incidentes históricos claves de la historia moderna de Guatemala e que ha influido negativamente en el desarrollo de la educación superior es el largo conflicto interno de 36 años. Navarrete y Acevedo (2009) coinciden en que "entre las consecuencias graves y menos consideradas del conflicto fue la dispersión $y$ el debilitamiento de la vida intelectual $y$ 
académica en Guatemala". Durante este período, el gobierno de Guatemala invirtió poco en la educación, se debilitaron considerablemente las estructuras culturales y educativas del país. La provisión de la educación superior se concentra en gran medida en el sector público. Para el año 2012 (véase la tabla 3) por lo menos 14 universidades están legalmente registradas y autorizadas para funcionar en el país, una pública y trece privadas. La USAC es altamente subsidiada por el Gobierno con una asignación constitucional del $5 \%$ de los ingresos públicos generales anuales. El resto de las universidades son instituciones privadas, junto con la universidad pública ofrece una cobertura del $7,05 \%$ de la población entre 15 y 34 años (Tobar, 2011).

Tabla 4 Matrícula en Educación Terciaria - Guatemala 2006-2010

\begin{tabular}{|l|l|l|l|l|l|l|l|l|}
\hline \multicolumn{2}{|l|}{} & \multicolumn{3}{c|}{$\%$} & & \multicolumn{3}{c|}{$\begin{array}{l}\text { Tasa de } \\
\text { Crecimiento }\end{array}$} \\
\hline Año & Total & Publica* & Privada & Publica & Privada & Total & Publica & Privada \\
\hline 2005 & 255,307 & 112,968 & 142,339 & 44.25 & 55.75 & 8.06 & -0.49 & 15.96 \\
\hline 2006 & 273,727 & 112,257 & 161,470 & 41.01 & 58.99 & 7.21 & -0.63 & 13.44 \\
\hline 2007 & 292,458 & 117,350 & 175,108 & 40.13 & 59.87 & 6.84 & 4.54 & 8.45 \\
\hline 2008 & 311,033 & 126,969 & 184,064 & 40.82 & 59.18 & 6.35 & 8.20 & 5.11 \\
\hline 2009 & 329,965 & 134,196 & 195,499 & 40.70 & 59.30 & 6.00 & 5.69 & 6.21 \\
\hline 2010 & 349,477 & 146,741 & 202,736 & 41.99 & 58.01 & 6.00 & 9.35 & 3.70 \\
\hline
\end{tabular}

Fuente: Tobar, 2011

La tabla 4 muestra la matrícula en la educación terciaria en Guatemala, tanto en universidades públicas como en las privadas. Es cierto que la cobertura ha crecido de manera constante, sin embargo, la cobertura de la educación terciaria en Guatemala evidencia el atraso en la formación de capital humano en el país. En 2012 había 312.697 estudiantes universitarios matriculados en el país, un porcentaje reducido de la población en riesgo en el grupo de edad entre 18-23 años de edad. Se calcula que la cobertura bruta de la educación superior en Guatemala es del $12 \%$, esto significaría 12 de todos los guatemaltecos con edades comprendidas entre 19-24 años de edad está inscrito. Esta es una de las más bajas de América Latina.

Steier y Yammal (2001: 11) también hacen hincapié en la gran concentración de la matrícula de educación superior de América Central y República Dominicana en los programas técnicos de grado y de pregrado. En cuanto a la educación de postgrado, la cobertura es muy baja, pocos programas de especialización y maestría están disponibles con sólo un puñado de programas de doctorado disponibles. Godínez y Tobar
(2006) también reconocen que no sólo los programas de postgrado son escasos, sino están demasiado concentrados en las ciencias sociales, las humanidades y las ramas del derecho.

Los indicadores en relación con los recursos humanos disponibles en Guatemala son escasos. Las instituciones públicas que se espera que produzcan indicadores tales como la Secretaría Nacional de Planificación y Programación de la Presidencia -SEGEPLAN-, la Secretaría Nacional de Ciencia y Tecnología-SENACYT-el Consejo Privado de Educación superior-CEPS-y la no generan suficiente información confiable integral.

Hay una percepción pública de que hay una gran escasez de RRHHAE en Guatemala, que fue confirmado por las respuestas obtenidas en la encuesta en línea. La Figura 2 muestra que sólo el $4 \%$ de los encuestados considera que hay demasiados o muchos RRHHAE en Guatemala, el 23\% considera que hay pocos RRHHAE en Guatemala. En contraste, un convincente $37 \%$ y $36 \%$, respectivamente, consideran que hay muy pocos o muy pocos RRHHAE en Guatemala. 
Figura 2 Disponibilidad de RRHHAE en Guatemala

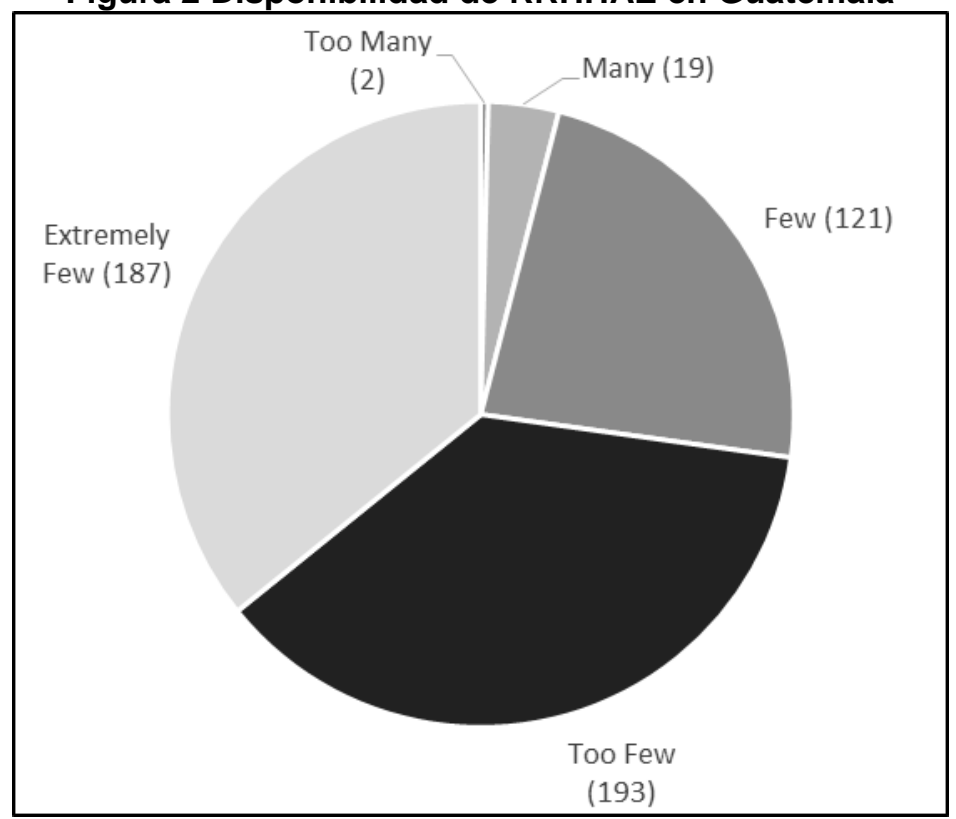

Note $n=522$

Los informantes clave entrevistados de los sectores público, privado y la sociedad civil, así como antiguos alumnos de los programas académicos de postgrado coinciden en que la cooperación internacional podría desempeñar un papel importante en el alivio de esta escasez de recursos humanos guatemaltecos con educación superior.

\section{b. Aprovechamiento de los Recursos Humanos Altamente Educados para el Desarrollo de Guatemala}

Distintas fuentes coinciden en que una de las deficiencias principales en Guatemala que dificulta la mejor utilización de RRHHAE es la falta de información y el registro sistemático. Esto es evidente en la ausencia de la producción de las estadísticas de contexto de trabajo, el diagnóstico y mapeo de recursos humanos según su capacitación y credenciales, (Sacayón, 2012; Tobar, 20111; Godínez y Tobar, 2006). Con frecuencia, en las evaluaciones regionales o mundiales de la educación superior, Guatemala aparece vacío o con información parcial (Brunner, 2011; UNESCO, 2012; UNESCO / IESALC, Schwartzaman, 2002). Periodista y otras fuentes (Hurtado,
2011; Menocal, 2006) están de acuerdo al señalar los niveles alarmantes de la improvisación debido a la insuficiente información disponible.

Otro problema que afecta negativamente el aprovechamiento de los RRHHAE en Guatemala es la injerencia omnipresente de la política en el sector académico. Diferentes fuentes han señalado que la Universidad de San Carlos de Guatemala juega al mismo tiempo los roles de regulador y regulado $y$ que por ello ha descuidado su papel crítico como el corazón de los académicos en Guatemala para convertirse en un actor político (Rodríguez, 2013; Hurtado, 2011; Torres, 2008). Progresivamente a lo largo de los años diferentes voces han insistido en la degradación de los académicos en la USAC, que han ido sustituyendo la academia por la política. Analistas consideran que la que en otros tiempos fuera una emblemática institución ha sido infiltrada por los partidos políticos, grupos de interés y dudosas prácticas clientelares (Castillo 2014; Maul, 2010).

Las responsabilidades que la Constitución de la República asigna a la única universidad pública incluyen la participación directa en al 
menos 44 instituciones de Gobierno. La Universidad de San Carlos ha sido calificada como "botín político" (Castillo, 2014) "ruinas académicas" (Fernández, 2010) y una "institución mediocre" (Mutilar, 2010).

Hay numerosos eventos electorales en esta universidad como la elección para asociaciones de estudiantes, a los decanatos, diferentes cargos de poder y la Rectoría Todas las elecciones se basan en las campañas que son prácticamente idénticas a las actividades realizadas por los partidos políticos a nivel nacional. De esta cuenta los recursos dedicados a la academia se reducen, las actividades educativas y de investigación escasean y se dedica parte importante de ellos a la política. Al mismo tiempo la deficiencia en la producción de la fuerza de trabajo altamente educada y necesaria para el desarrollo de Guatemala está en suspenso.

Mientras que la producción de RRHHAE en Guatemala es limitada, el sector de empleo que mejor hace uso de los guatemaltecos con educación superior para el desarrollo tal país también fue un elemento central del presente estudio. Los participantes de la encuesta en línea respondieron a la siguiente pregunta:

¿En su opinión qué sector del contexto de trabajo de Guatemala aprovecha mejor los recursos humanos guatemaltecos altamente educados para el desarrollo del país?

La Figura 3 resume las respuestas. Según los datos recogidos, el $48 \%$ de los encuestados considera que el sector privado, entendida como organizaciones que buscan negocios orientados a la ganancia, es el área del contexto de trabajo en Guatemala que mejor utiliza los RRHHAE. El siguiente sector señalado fue la academia con un $24 \%$ y la sociedad civil o las organizaciones no gubernamentales con un 13\%. La última opción en la opinión de los encuestados fue el sector público con un 10\%. La opción otros sectores fue seleccionado por un $5 \%$.

Figura 3 Sector según contexto de trabajo en Guatemala que mejor utilice RRHHAE para el desarrollo del país

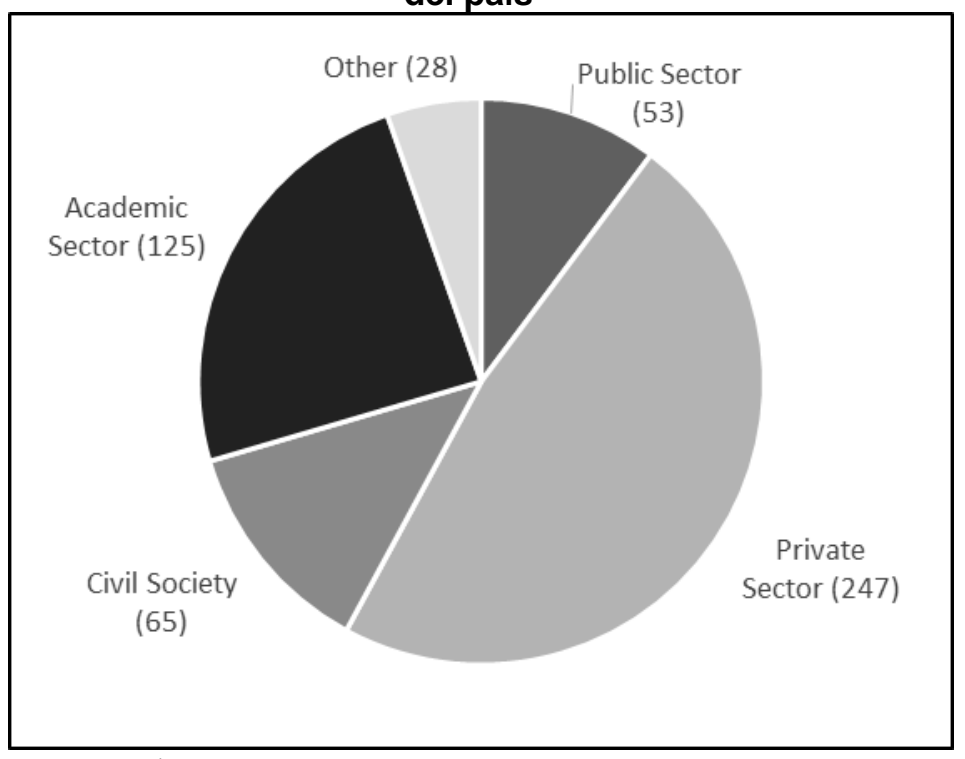

Nota: $n=518$ 
Los datos cualitativos también se recogieron para explicar las razones que explican la selección realizada por los encuestados con respecto al sector del contexto de trabajo que mejor utiliza los recursos humanos guatemaltecos con educación superior. Los datos fueron ordenados y clasificados en dos temas principales: a. Condiciones para Aprovechamiento de RRHHAE y b. Desafíos para el aprovechamiento de RRHHAE.

Las tablas 5 y 6 resumen las respuestas en las que diferentes aspectos de la cultura del trabajo por sectores se citan por los encuestados como responsables del mayor o menor aprovechamiento del recurso humano altamente educado que a su vez es valioso porque se forma a pesar de las deficiencias del sistema de educación superior en Guatemala. El sector privado y la academia son los sectores seleccionados por la mayoría porque tienden a utilizar los
RRHHAE en Guatemala, aunque esta segunda se resalta como la que más puede maximizar los beneficios para el desarrollo de Guatemala, en términos de los beneficios y rentabilidad social.

Por un lado, las competencias adquiridas en la educación de postgrados, incluidas la investigación avanzada, la innovación, y la creación de redes podría mejorar la aplicación de buenas prácticas y conocimientos, particularmente en la enseñanza superior y la producción de nuevos conocimientos. Esto último ha demostrado ser un problema acuciante en Guatemala, toda vez que el sistema de educación superior no ha sido capaz de producir la fuerza de trabajo crítico y con talento suficiente.

Tabla 5 Aprovechamiento de los RRHHAE guatemaltecos para el desarrollo del país

\begin{tabular}{|l|l|}
\hline $\begin{array}{l}\text { Sector de } \\
\text { Trabajo }\end{array}$ & $\begin{array}{l}\text { Razones que justifican el mejor aprovechamiento de los RRHHAE en } \\
\text { Guatemala }\end{array}$ \\
\hline $\begin{array}{l}\text { Sector } \\
\text { público }\end{array}$ & $\begin{array}{l}\text { - Posiciones estables permiten a los graduados implementar en sus instituciones } \\
\text { nuevo conocimiento } \\
\text {-Instituciones públicas sirven a la población, por tanto, el impacto de los } \\
\text { guatemaltecos altamente educados es más alto }\end{array}$ \\
\hline $\begin{array}{l}\text { Sector } \\
\text { privado }\end{array}$ & $\begin{array}{l}\text {-El reclutamiento es con base a méritos y la contratación es más eficiente } \\
\text {-Tiene más recursos disponibles para los proyectos y programas } \\
\text {-Valora más la educación y estimula la innovación }\end{array}$ \\
\hline $\begin{array}{l}\text { Sector } \\
\text { académico }\end{array}$ & $\begin{array}{l}\text {-Es más probable que mantenga el desarrollo académico y lo replique } \\
\text {-Se valoran las habilidades de investigación } \\
\text {-El impacto de la transferencia de conocimiento es mayor en las tareas docentes } \\
\text { en lugar de la administración pública o la búsqueda de lucro } \\
\text {-La universidad es un empleador más estable y una educación de buena calidad } \\
\text { es profundamente necesaria en el país }\end{array}$ \\
\hline Sociedad civil & $\begin{array}{l}\text {-Posibilidad para retorno social de estudios de postgrado } \\
\text {-La sociedad civil proporciona servicios que tanto el sector público como el } \\
\text { privado no están cumpliendo } \\
\text { - Permiten desarrollar una carrera }\end{array}$ \\
\hline
\end{tabular}

Fuente: Compilado por los autores de las respuestas recurrentes de los participantes

Estos resultados son particularmente interesantes ya que varios países donantes privilegian el apoyo a candidatos para becas para estudios de postgrado que sean propuestos por las instituciones públicas. Aunque conceptualmente el objetivo de contribuir a la profesionalización de los funcionarios públicos puede estar bien orientado, las condiciones ofrecidas por el país socio, en este caso Guatemala, deben ser consideradas con el fin de contribuir eficazmente al mejor aprovechamiento de los recursos humanos guatemaltecos con educación superior. 


\begin{tabular}{|c|c|}
\hline $\begin{array}{l}\text { Sector de } \\
\text { trabajo }\end{array}$ & $\begin{array}{l}\text { Retos al aprovechamiento de los RRHHAE guatemaltecos para el desarrollo del } \\
\text { país }\end{array}$ \\
\hline $\begin{array}{l}\text { Sector } \\
\text { público }\end{array}$ & $\begin{array}{l}\text { - Las habilidades técnicas y el conocimiento sofisticado no son aplicables } \\
\text {-El nepotismo y las conexiones personales son más importantes que los méritos y la } \\
\text { educación } \\
\text { - La falta de planificación y visión a largo plazo } \\
\text { - La educación no es importante para convertirse en un funcionario público, sólo las } \\
\text { conexiones políticas y el activismo electoral } \\
\text { - Las instituciones públicas discriminan a quienes han obtenido títulos de postgrado } \\
\text { nacionales y más aún en el extranjero } \\
\text {-Desincentivos, salarios pobres, la inestabilidad, no hay carrera civil administrativa } \\
\text {-Interés específico de mantener a las instituciones públicas con personal de bajo } \\
\text { nivel educativo }\end{array}$ \\
\hline $\begin{array}{l}\text { Sector } \\
\text { privado }\end{array}$ & $\begin{array}{l}\text { - El espíritu empresarial se enfrenta a duras condiciones como medios de producción } \\
\text { totalmente concentrados en pocas manos } \\
\text {-Los empresas privadas en Guatemala sólo están interesadas en las prácticas } \\
\text { extractivas no en el desarrollo del país }\end{array}$ \\
\hline $\begin{array}{l}\text { Sector } \\
\text { académico }\end{array}$ & $\begin{array}{l}\text {-No hay investigación en la práctica del sector académico de Guatemala } \\
\text { - Universidades privadas proporcionan más oportunidades para innovar, la } \\
\text { educación superior pública en Guatemala es extremadamente politizada y corrupta } \\
\text {-Magísteres y doctores graduados compiten para cargos de profesores con títulos } \\
\text { sólo a nivel de licenciatura, irónicamente estos últimos obtienen las posiciones }\end{array}$ \\
\hline Sociedad civil & $\begin{array}{l}\text {-Sólo ONG internacionales aprecian a los guatemaltecos con talento, las ONG } \\
\text { locales son similares a los partidos políticos } \\
\text {-Existe la percepción equivocada de que los guatemaltecos altamente educados no } \\
\text { desean trabajar en este sector }\end{array}$ \\
\hline
\end{tabular}

Fuente: Compilado por los autores de las respuestas recurrentes de los participantes

Los años de crecimiento desordenado y la falta de revisiones de planificación, evaluación y calidad han creado un sistema de educación superior caótico y fragmentado. Esto ha afectado negativamente la calidad; la pertinencia de la educación superior en Guatemala también está en riesgo. Teniendo en cuenta los elementos propuestos por Steiner y Yammal (2001) la calidad de la educación terciaria se puede entender en cuatro dimensiones: i. Características de los profesores y miembros de la facultad, ii. Desarrollo Curricular, iii. Características de los alumnos de nuevo ingreso, y iv. Existencia de mecanismos de garantía de calidad. Guatemala puntea bajo en las cuatro.

Una vez más, la falta de información dificulta el análisis del nivel de formación y áreas de especialización de los profesores que se dedican a la educación superior tanto pública como privada. Gutiérrez y De la Rosa (2004) estiman que sólo un $10 \%$ de los profesores de tiempo completo en la Universidad de San Carlos de Guatemala tiene títulos de postgrado (en El Salvador el porcentaje es del $27 \%$ ), mientras que los docentes con doctorado son escasos. Sólo el 9,2\% del cuerpo docente tiene dedicación completa, mientras que el $90,2 \%$ restante son ya sea contratados a tiempo parcial o son profesores contratados temporalmente. Se estima que más del $75 \%$ de los profesores universitarios trabajan por horas. Esto contrasta con Costa Rica, país que cuenta con un $67 \%$ de los profesores de tiempo completo.

Un énfasis generalizado en las actividades exclusivas de enseñanza como propósito educativo perpetúa la reproducción del conocimiento en lugar de la producción de nuevos conocimientos; la investigación, la ciencia y la tecnología son campos aún no explorados a profundidad $y$, de manera generalizada, no se refleja como prioridad en las instituciones de educación superior 
(Hernández y Blas, 2013). En comparación con otros países de América Latina, la investigación académica y la producción científica producida en Guatemala es escasa. No sólo la educación superior es débil en la cobertura y la accesibilidad a la mayoría de la población en Guatemala sino también está muy concentrada en las ciencias sociales. En las tablas 7 y 8 se resume el número de graduados del sistema nacional de educación superior, de maestría y doctorado.

Tabla 7 Graduados de maestría de las instituciones locales en Guatemala (2001-2011)

\begin{tabular}{|c|c|c|c|c|c|c|c|c|c|c|c|}
\hline 2001 & 2002 & 2003 & 2004 & 2005 & 2006 & 2007 & 2008 & 2009 & 2010 & 2011 & Campo \\
\hline 1 & 4 & 2 & 15 & 1 & sd & sd & 21 & 21 & 4 & 7 & Ciencias Natural y Exactas \\
\hline 32 & 35 & 44 & 41 & 38 & 64 & 185 & 150 & 200 & 118 & 275 & Ingeniería y Tecnología \\
\hline 181 & 188 & 234 & 200 & 54 & 52 & 142 & 75 & 307 & 415 & 407 & Ciencias Médicas \\
\hline sd & sd & sd & 2 & 15 & 47 & 86 & 93 & 40 & 26 & 10 & Ciencias Agrícolas \\
\hline 141 & 141 & 292 & 322 & 535 & 799 & 822 & 827 & 942 & 1572 & 1334 & Ciencias Sociales \\
\hline 31 & 23 & 26 & 32 & 54 & 12 & 22 & 30 & 100 & 77 & 60 & Humanidades \\
\hline 386 & 391 & 598 & 612 & 697 & 974 & 1257 & 1196 & 1610 & 2212 & 2093 & Total \\
\hline
\end{tabular}

Fuente: RYCIT (2013) http://www.ricyt.org/ sd= sin datos

Como se puede observar, las ciencias sociales concentraron el $60 \%$ de los graduados, en el caso de magísteres, desde 2001-2011, seguido por Ciencias Médicas, e Ingeniería y Tecnología. El caso de los títulos de doctorado es aún más grave en términos del bajo número de graduados y el alto nivel de concentración en las ciencias sociales con un $91 \%$. De 140 alumnos de las instituciones nacionales que concedieron el doctorado entre 2001 y 2011 un total de 128 concentrados en ciencias sociales equivalente al $91 \%$ del total.

Tabla 8 Graduados de doctorado de las instituciones locales en Guatemala (2001-2011)

\begin{tabular}{|r|r|r|r|r|r|r|r|r|r|r|l|}
\hline 2001 & 2002 & 2003 & 2004 & 2005 & 2006 & 2007 & 2008 & 2009 & 2010 & 2011 & Campo \\
\hline & & & & & & & & & & & Ciencias Exactas y Naturales \\
\hline & & & & 2 & & & & & & & Ingenierías y Tecnología \\
\hline & & & & & 2 & 1 & 2 & & & & Ciencias Médicas \\
\hline & & & & & & & & & & & Ciencias Agrícolas \\
\hline 3 & & 3 & 6 & 9 & 16 & 8 & 5 & 25 & 16 & 37 & Ciencias Sociales \\
\hline & 1 & & & & & 1 & 3 & & & & Humanidades \\
\hline 3 & 1 & 3 & 6 & 11 & 18 & 10 & 10 & 25 & 16 & 37 & Total \\
\hline
\end{tabular}

Fuente: RYCIT (2013) http://www.ricyt.org/ 
Esta falta de diversificación de los campos académicos observados en el sistema de educación superior interno de Guatemala es preocupante ya que la innovación y las condiciones laborales son más probables de ocurrir con una fuerza de trabajo crítico sólida en la ciencia, la ingeniería y campos técnicos. En un país con necesidades abundantes podría parecer que todos los campos académicos y profesionales están en la máxima prioridad con el fin de llevar a Guatemala por el camino del desarrollo. La encuesta en línea solicita a los mismos encuestados asignar un nivel de prioridad a ocho campos académicos que van desde 1 a la más alta prioridad y 8 para la más baja.

Tabla 9 Prioridad académica/campo profesional para el desarrollo de Guatemala

\begin{tabular}{|l|r|r|r|r|c|c|c|c|}
\hline Campo Académico/Nivel de Prioridad & $\mathbf{1}$ & $\mathbf{2}$ & $\mathbf{3}$ & $\mathbf{4}$ & $\mathbf{5}$ & $\mathbf{6}$ & $\mathbf{7}$ & $\mathbf{8}$ \\
\hline Ciencias Sociales & 144 & 87 & 63 & 44 & 39 & 45 & 49 & 45 \\
\hline $\begin{array}{l}\text { Educación, Humanidades y Ciencias del } \\
\text { Comportamiento }\end{array}$ & 214 & 76 & 50 & 29 & 30 & 39 & 30 & 48 \\
\hline Administración, Negocios, Economía y Finanzas & 103 & 108 & 95 & 53 & 54 & 31 & 30 & 42 \\
\hline Ciencias Naturales y Exactas & 113 & 95 & 85 & 63 & 37 & 43 & 30 & 50 \\
\hline Biotecnología, Ciencias del Agro y de Ambiente & 163 & 100 & 76 & 35 & 34 & 32 & 31 & 45 \\
\hline Ingeniería y Tecnología & 193 & 82 & 61 & 37 & 31 & 28 & 41 & 43 \\
\hline Ciencias Médicas y de la Salud & 233 & 93 & 46 & 30 & 20 & 20 & 25 & 49 \\
\hline Administración Pública y Relaciones Internacionales & 110 & 83 & 80 & 57 & 49 & 41 & 42 & 49 \\
\hline
\end{tabular}

Fuente: RYCIT (2013) http://www.ricyt.org/

\section{Conclusiones}

Los RRHHAE son más necesarios cada día para las sociedades que aspiran a tomar parte en el nuevo paradigma de la economía del conocimiento. Guatemala necesita desesperadamente multiplicar sus esfuerzos para acumular una fuerza de trabajo calificada y bien preparada, capaz de responder a las crecientes presiones de la globalización y los paradigmas productivos cambiantes. Es preocupante que el sistema de educación superior nacional en Guatemala ha demostrado ser insuficiente para producir la fuerza de trabajo educada avanzada que se necesita.

El debate de la educación en Guatemala todavía se concentra en la educación primaria. Los desafíos que se enfrentan en este nivel son utilizados por varias autoridades y pensadores para desviar la atención de la educación terciaria; sin embargo, la educación superior ya no se puede descuidar. Este escenario da la bienvenida a la contribución del apoyo de los donantes para aliviar la escasez de recursos humanos altamente calificados Guatemala. Desde la década de los 80 s jóvenes líderes y prometedores han sido educados en el nivel de postgrado con la valiosa contribución de la comunidad internacional; se espera que el país de origen, en este caso Guatemala, incorporare a los graduados en las diferentes áreas de trabajo con el fin de hacer el mejor uso de estos recursos humanos altamente educados y escasos.

Los datos recogidos y analizados en este artículo deben considerarse en el contexto de los resultados del sistema de educación superior. Como en esta investigación se definen los "recursos humanos guatemaltecos altamente educados" como a individuos que han completado la formación de postgrado (master / doctorado), se presupone como requisito previo para que los candidatos a ser aceptados en los programas académicos de postgrado que han previamente obtenido un título universitario de licenciatura. Por eso, la estructura, la cobertura, la calidad y el estado general de la educación superior en 
Guatemala determinan en un grado importante las características de los individuos que persiguen niveles más avanzados de educación.

Sobre la base de los datos y la información disponible, se puede concluir que los recursos humanos altamente calificados guatemaltecos son escasos. Incluso si consideramos todos los exalumnos de postgrado, tanto de universidades nacionales y aún más de universidades internacionales, la escasez de mano de obra calificada y educada y avanzada está socavando gravemente la competitividad del país. Por otro lado, la producción de tan valioso capital humano es compleja y consume muchos recursos. Las universidades locales a nivel de postgrado no facilitan el acceso a la financiación pública, ni privada, ni siquiera la Universidad de San Carlos de Guatemala. En Guatemala, todos los programas de educación de postgrado son financiados por los propios estudiantes. El caso de la educación superior privada es aún más agudo pues el lucro, la búsqueda de beneficios privados y la falta de préstamos educativos contribuye a la exclusión y sólo las élites económicas tienen acceso a la educación superior a este nivel (Roma, 2006; Godínez y Tobar, 2006).

Los hallazgos de este estudio indican que los negocios privados en Guatemala y la academia (universidades y centros de investigación) son considerados por los participantes como los sectores de la sociedad guatemalteca que más valoran y aprovechan el recurso humano altamente educado; contrariamente, la percepción del sector público enfatiza en dejar de lado la importancia de los recursos humanos para el desarrollo nacional. Los encuestados proporcionan diferentes razones para explicar su percepción. El sector privado se percibe como más "meritocrático y basado en la capacidad", sin embargo, la prevalencia del interés de lucro, y, posiblemente, la captación de rentas, también se percibe como elemento disuasorio para el desarrollo del país.
El sistema de educación superior en Guatemala necesita desarrollar un serio esfuerzo para superar retos fundamentales y frente a las múltiples carencias que sufre, principiando por la falta de información y registros consistentes de cobertura nacional. Es cierto que la Universidad de San Carlos de Guatemala ha puesto en marcha la Dirección de Registro y Estadística, a cargo, al menos, de documentar la inscripción de los estudiantes y las estadísticas generales. Al mismo tiempo, la universidad pública ha creado diferentes unidades encargadas de recabar la información sobre los profesores, empleados, infraestructura, de pregrado y oferta académica de postgrado; sin embargo, la información consolidada no está disponible.

El caso es tan desalentador que entre las instituciones privadas de educación superior no es muy diferente. Cada universidad es un ente aislado y autónomo, se conocen esfuerzos incipientes por sistematizar indicadores sin coordinación alguna. La fragmentación del sistema de educación superior dificulta cualquier intento de desarrollar un diagnóstico de la disponibilidad de recursos humanos guatemaltecos con educación superior.

La situación es aún más crítica para las personas educadas en el extranjero. Las estimaciones (Arenas, 2012) indican que sólo una pequeña minoría de graduados de universidades extranjeras sigue los procedimientos para incorporar sus grados académicos obtenidos en el extranjero.

Durante años, el debate sobre la asignación de recursos escasos (desde la cooperación pública, privada internacional, etc.) Para resolver los acuciantes problemas en todos los niveles de la educación se han orientado a la educación primaria y secundaria. En este punto, la educación superior y su enorme responsabilidad de formar la mano de obra calificada para las necesidades inmediatas de la economía y la sociedad no se puede retrasar más.

El sector académico tiene una urgente necesidad de fortalecer la calidad del 
personal administrativo, los investigadores, incluso los profesores y miembros del cuerpo docente. Al mismo tiempo, los beneficios sociales de la incorporación de los recursos humanos guatemaltecos con educación superior podrían ser más amplios y más profundos. El caso de la Universidad de San Carlos de Guatemala es particularmente crítico. Como la única universidad pública, se espera que esta institución facilite el acceso a la educación superior para el pueblo de Guatemala que no pueden pagar la educación privada o en el extranjero. Sin embargo, esta misión no se ha cumplido todavía, debido a varios retos y dificultades, como la gobernanza, las limitaciones financieras y la interferencia política.

Por lo tanto, la solución a medio plazo de la actual situación del estancamiento del caso guatemalteco se puede realizar en dos frentes: En primer lugar, la educación superior nacional necesita una reforma seria para la adopción de un entorno competitivo en el sistema universitario público. En segundo lugar, al maximizar el uso del sistema de formación en el extranjero ofrecido con apoyo de países donantes, las deficiencias actuales en el sistema de educación superior podrían abordarse, es decir, tener más profesores educados a nivel de doctorado, la integración de los niveles más altos de campos académicos, tanto vertical (aumentar la oferta de programas de postgrado en las universidades) como horizontalmente (diversificación campos académicos).

\section{Recomendaciones}

Este trabajo intenta contribuir al debate de la disponibilidad y el mejor uso de los recursos humanos guatemaltecos con educación superior. Algunos investigadores constantemente citan artículos de publicaciones en prensa escrita (Sacayón, 2012; Godínez y Tobar, 2006) pues se padece la falta de investigación académica en Guatemala. En este sentido, este estudio anima a los responsables políticos, las autoridades de educación superior e investigadores a la construcción de un amplio cuerpo de literatura que trate los temas más urgentes relacionados con la educación superior, los RRHHAE y la cooperación internacional. Por esta, entre otras razones, los valiosos recursos de la cooperación de desarrollo dedicado al financiamiento de estudios de postgrado de guatemaltecos potenciales líderes es de mayúscula importancia.

Todavía existen pocos estudios sobre los vínculos entre la educación superior y los problemas socioeconómicos en Guatemala. Generar información básica y los estudios sistemáticos que aborden la producción y gestión de los recursos humanos en Guatemala es una tarea pendiente. El sentido común indica que la información es poder, la toma de decisiones exige estadísticas fiables, diagnósticos y planes de trabajo.

Otro tema importante a ser explorado es el papel desempeñado por los donantes internacionales que apoyan para abordar los desafíos actuales o complementar los esfuerzos existentes para educar a un grupo de personas altamente capacitadas. El desarrollo de la capacidad humana en Guatemala por medio de la educación de postgrados en el extranjero financiados por la ayuda extranjera ha estado en vigor desde la década de 1970. Japón fue uno de los primeros donantes para ofrecer oportunidades de estudios de postgrado a los guatemaltecos con talento. Se invitó a los primeros alumnos de becas para estudiar Medicina en 1974 (Embajada de Japón, 2013). Desde entonces otros países donantes han adoptado un enfoque similar a cooperar con Guatemala, por ejemplo, el Programa Fulbright de Estados Unidos en 1984, Alemania y el DAAD en 1985, y Holanda y el NFP-NUFFIC, así como el MAEC-AECID de España en la década de 1990. Más tarde, más países se unieron, como el Reino Unido con el Programa Chevening, la República de Corea con el Programa de Becas de KOICA, Taiwán y la IDCF y México y su programa de Becas para Extranjeros desde mediados de los 2000. Esfuerzos serios de investigación deberán ser destinados a estudiar el impacto en el desarrollo de capacidades a través de esta oferta becaria. 


\section{Referencias}

Aldana, Carlos, entrevista de Kleinsy Bonilla. Former Viceminister of Education (13 de February de 2014).

Arenas, Luis Fernando, entrevista de Kleinsy Bonilla. Director General of the Institute for the Development of Superior Education in Guatemala (15 de november de 2012).

Brunner, Jorge Joaquin \& Rocio Ferrada Hurtado. Educacion Superior en Iberoamerica [Superior education in Latin America]. Informe, Santiago: Centro Universitario de Desarrollo CINDA, 2011.

Calderon, Jose Humberto. Estudio sobre la Repitencia y Desercion en la Educacion Superior de Guatemala [Study about Repeating and Abandonment in Superior Education of Guatemala]. Guatemala City: UNESCO-IESALC, 2005.

Castillo, Juan Manuel. «Preciado Botin [Precious loot].» Prensa Libre, 14 de February de 2014: 6.

Chamarbagwala, Rubiana \& Hilcias E. Moran. «The Human Capital Consequencesof CivilWar: Evidence from Guatemala.» Journal of Development Economics 94 (2010): 41-61.

Retos y Alternativas de la Educacion Superior en Guatemala [Challenges and Alternatives of Superior Education in Guatemala]. Dirigido por Fernando Arredondo. Interpretado por Olmedo Espana. 2009.

Fernandez, Dina. La USAC en Piltrafas [Ramainings of USAC] . Guatemala City, 21 de September de 2013.

Fuentes, Juan Alberto, entrevista de Kleinsy Bonilla. Former Minister of Finance of Guatemala 2008-2010 (26 de 07 de 2013).

FUNDESA ( Foundation for the Development of Guatemala). «Economic Perspectives for Guatemala, 2013.» Guatemala, City: FUNDESA, 21 de May de 2012.

Galvez, Estuardo. "La USAC es el Reflejo de la Sociedad.» Prensa Libre, 09 de 07 de 2006: 1617.

Godinez, Maria del Rosario, Luis Alfredo Tobar Piril. Los Estudios de Postgrado en la Universidad de San Carlos de Guatemala [Postgraduate
Education in San Carlos ofGuatemala University]. Report, Guatemala City: USAC/IESAL-UNESCO, 2006.

Gonzalez, Ana Lucia. «Por que tan pocos Becarios? [Why so few scholarship fellows?].» Revista D. Guatemala City, Guatemala: Prensa Libre, 17 de November de 2013.

Gutierrez Herrera, William Roberto and Francisco Bernardo Raul de la Rosa. Perfil del Profesor Universitario en el Area de Matematicas en la Republica de Guatemala [Profile of the University Professor in the Area of MAtematics in the Republic of Guatemala]. Guatemala City: San Carlos of Guatemala University, School of Engineering, 2004.

Hernandez, Violeta and Ana Lucia Blas. Vinculos entre Universidades y Think Tanks: Una Propuesta [Links between Universities and Think Tanks: A Proposal]. Guatemala City: Saber America Latina, 2013.

Hurtado, Paola. «Los 312,697 Universitarios Ignotos [The 312,697 unknown university students].» El Periodico, 03 de 04 de 2011: 7-11.

Iteriano, Elder. «Guatemala, de los mas rezagados en educacion en Latinoamerica [Guatemala, among the most backwarness countries in Education in Latin America].» Siglo 21, 18 de 12 de 2012: 11 .

Knut, Walter. La Educacion en CentroAmerica: Reflexiones en Torno a sus Problemas y Ptencial [Education in Central America: Reflections about its problems and potential]. Working Paper, Hamburg: Institute for IberoAmerica, 2009.

Leon, Susana de. «De Guatemala para el Mundo: Mentes que Brillan [From Guatemalan to the World, Brillian Brains].» El Periodico, 17 de 02 de 2013: 3-5.

Maul, Hugo. "USAC: Triste Destino [USAC: Sad Destiny].» El Periodico, 22 de February de 2010: 8.

Medina, Efrain, entrevista de Kleinsy Bonilla. Former Rector of San Carlos of Guatemala University (11 de February de 2014).

Menocal, Carlos. "Crisis en la USAC Genera pocos Graduados.» Prensa Libre, 30 de april de 2006: 2-4. 
Ministry of Foreign Affairs of Japan. Study in Japan. 06 de 06 de 2013. http://www.studyjapan.go.jp/en/faq/faq10e.html (último acceso: 09 de 08 de 2013).

Moreno, Juan Carlos \& Pablo Ruiz. La Educacion Superior y el Desarrollo Economico de America Latina [Higher education and economic development in Latin America]. Serie Estudios y Perspectivas, Mexico, D. F.: United Nations Comission for Latin America CEPAL, 2009.

Parrilla, Ekaterina, entrevista de Kleinsy Bonilla. National Secretary for Planning and Programming of the Presidency of Guatemala SEGEPLAN (04 de May de 2013).

Ponce Villela, Edgar Augusto and Amilcar Folgar Portillo. Caracterizacion de la Cultura Organizacional de la Universidad de San Carlos de Guatemala [Organizational Culture San Carlos of Guatemala University]. Institutional Report, Guatemala City: San Carlos of Guatemala University, 2007.

Rama, Claudio. "Los Postgrados en America Latina en la Sociedad del Saber [Postgraduate education in Latin America in the knolwdege society].» En Informe sobre la Educacion Superior en America Latina y El Caribe 2000-2005 [Report about Superior Education in Latin America and the Caribbean2000-2005], de UNESCO/IESALC, 4355. Caracas: Editorial Metamorfosis C.A., 2006.

Rubio, Miriam, entrevista de Kleinsy Bonilla. National Secretary of Sciece and Technology (2013 de October de 2013).

Sacayon, Eduardo Enrique. Los Sistemas de Acceso, Normativas de Permanencia y Estrategias de Tutoria y Retencion de Estudiantes de Educacion Superior, Informe Nacional Guatemala [Systems for acess, regulations, permanece and strategies of tutoring and retaining superior education]. Guatemala City: Institute for Interethnic Studies, San Carlos of Guatemala University, 2012.

Schwartzaman, Simon. Higher Education and the Demands of the New Economy in Latin America. Background Paper, Latin American and Caribbean Department, Sao Paulo: World Bank, 2002.

Steier, Francis and Cesar Yammal. La Educacion Superior en Centro America y Republica Dominicana [Superior Education in Central America and Dominican Republic]. Discussion paper for the Conference in Superior Education held in Guatemala, May 30-June 1st. 2001, Antigua Guatemala: World Bank, 2001.

The Task Force on Higher Education and Society. Higher Education in Developing Countries. Peril and Promise. Washington: The World Bank, 2000.

Tobar, Luis Alfredo. «La Educacion Superior en Guatemala en la Primera Decada del Siglo XXI [Superior Education in Guatemala during the first decade of XXI century]." Revista Innovacion Educativa 11, ํㅡ 57 (2011): 69-80.

Torres, Milton Alfredo. «Los Politiqueros se Aduenan de la USAC [Political actors become owners of USAC].» La Hora, 19 de February de 2008: 8 .

Aldana, Carlos, entrevista de Kleinsy Bonilla. Former Viceminister of Education (13 de February de 2014).

Arenas, Luis Fernando, entrevista de Kleinsy Bonilla. Director General of the Institute for the Development of Superior Education in Guatemala (15 de november de 2012).

Brunner, Jorge Joaquin \& Rocio Ferrada Hurtado. Educacion Superior en Iberoamerica [Superior education in Latin America]. Informe, Santiago: Centro Universitario de Desarrollo CINDA, 2011.

Calderon, Jose Humberto. Estudio sobre la Repitencia y Desercion en la Educacion Superior de Guatemala [Study about Repeating and Abandonment in Superior Education of Guatemala]. Guatemala City: UNESCO-IESALC, 2005.

Castillo, Juan Manuel. «Preciado Botin [Precious loot].»Prensa Libre, 14 de February de 2014: 6.

Chamarbagwala, Rubiana \& Hilcias E. Moran. "The Human Capital Consequencesof CivilWar: Evidence from Guatemala.» Journal of Development Economics 94 (2010): 41-61.

Retos y Alternativas de la Educacion Superior en Guatemala [Challenges and Alternatives of Superior Education in Guatemala]. Dirigido por Fernando Arredondo. Interpretado por Olmedo Espana. 2009.

Fernandez, Dina. La USAC en Piltrafas [Ramainings of USAC] . Guatemala City, 21 de September de 2013. 
Fuentes, Juan Alberto, entrevista de Kleinsy Bonilla. Former Minister of Finance of Guatemala 2008-2010 (26 de 07 de 2013).

FUNDESA ( Foundation for the Development of Guatemala). «Economic Perspectives for Guatemala, 2013.» Guatemala, City: FUNDESA, 21 de May de 2012.

Galvez, Estuardo. "La USAC es el Reflejo de la Sociedad.»Prensa Libre, 09 de 07 de 2006: 1617.

Godinez, Maria del Rosario, Luis Alfredo Tobar Piril. Los Estudios de Postgrado en la Universidad de San Carlos de Guatemala [Postgraduate Education in San Carlos ofGuatemala University]. Report, Guatemala City: USAC/IESAL-UNESCO, 2006.

Gonzalez, Ana Lucia. "Por que tan pocos Becarios? [Why so few scholarship fellows?].» Revista D. Guatemala City, Guatemala: Prensa Libre, 17 de November de 2013.

Gutierrez Herrera, William Roberto and Francisco Bernardo Raul de la Rosa. Perfil del Profesor Universitario en el Area de Matematicas en la Republica de Guatemala [Profile of the University Professor in the Area of MAtematics in the Republic of Guatemala]. Guatemala City: San Carlos of Guatemala University, School of Engineering, 2004.

Hernandez, Violeta and Ana Lucia Blas. Vinculos entre Universidades $y$ Think Tanks: Una Propuesta [Links between Universities and Think Tanks: A Proposal]. Guatemala City: Saber America Latina, 2013.

Hurtado, Paola. «Los 312,697 Universitarios Ignotos [The 312,697 unknown university students].» El Periodico, 03 de 04 de 2011: 7-11.

Iteriano, Elder. «Guatemala, de los mas rezagados en educacion en Latinoamerica [Guatemala, among the most backwarness countries in Education in Latin America].» Siglo 21, 18 de 12 de 2012: 11.

Knut, Walter. La Educacion en CentroAmerica: Reflexiones en Torno a sus Problemas y Ptencial [Education in Central America: Reflections about its problems and potential]. Working Paper, Hamburg: Institute for IberoAmerica, 2009.
Leon, Susana de. «De Guatemala para el Mundo: Mentes que Brillan [From Guatemalan to the World, Brillian Brains].» El Periodico, 17 de 02 de 2013: 3-5.

Maul, Hugo. "USAC: Triste Destino [USAC: Sad Destiny].» El Periodico, 22 de February de 2010: 8.

Medina, Efrain, entrevista de Kleinsy Bonilla. Former Rector of San Carlos of Guatemala University (11 de February de 2014).

Menocal, Carlos. "Crisis en la USAC Genera pocos Graduados.» Prensa Libre, 30 de april de 2006: 2-4.

Ministry of Foreign Affairs of Japan. Study in Japan. 06 de 06 de 2013. http://www.studyjapan.go.jp/en/faq/faq10e.html (último acceso: 09 de 08 de 2013).

Moreno, Juan Carlos \& Pablo Ruiz. La Educacion Superior y el Desarrollo Economico de America Latina [Higher education and economic development in Latin America]. Serie Estudios y Perspectivas, Mexico, D. F.: United Nations Comission for Latin America CEPAL, 2009.

Parrilla, Ekaterina, entrevista de Kleinsy Bonilla. National Secretary for Planning and Programming of the Presidency of Guatemala SEGEPLAN (04 de May de 2013).

Ponce Villela, Edgar Augusto and Amilcar Folgar Portillo. Caracterizacion de la Cultura Organizacional de la Universidad de San Carlos de Guatemala [Organizational Culture San Carlos of Guatemala University]. Institutional Report, Guatemala City: San Carlos of Guatemala University, 2007.

Rama, Claudio. "Los Postgrados en America Latina en la Sociedad del Saber [Postgraduate education in Latin America in the knolwdege society]." En Informe sobre la Educacion Superior en America Latina y El Caribe 2000-2005 [Report about Superior Education in Latin America and the Caribbean2000-2005], de UNESCO/IESALC, 4355. Caracas: Editorial Metamorfosis C.A., 2006.

Rubio, Miriam, entrevista de Kleinsy Bonilla. National Secretary of Sciece and Technology (2013 de October de 2013).

Sacayon, Eduardo Enrique. Los Sistemas de Acceso, Normativas de Permanencia y Estrategias 
de Tutoria y Retencion de Estudiantes de Educacion Superior, Informe Nacional Guatemala [Systems for acess, regulations, permanece and strategies of tutoring and retaining superior education]. Guatemala City: Institute for Interethnic Studies, San Carlos of Guatemala University, 2012.

Schwartzaman, Simon. Higher Education and the Demands of the New Economy in Latin America. Background Paper, Latin American and Caribbean Department, Sao Paulo: World Bank, 2002.

Steier, Francis and Cesar Yammal. La Educacion Superior en Centro America y Republica Dominicana [Superior Education in Central America and Dominican Republic]. Discussion paper for the Conference in Superior Education held in Guatemala, May 30-June 1st. 2001, Antigua Guatemala: World Bank, 2001.

The Task Force on Higher Education and Society. Higher Education in Developing Countries. Peril and Promise. Washington: The World Bank, 2000.

Tobar, Luis Alfredo. «La Educacion Superior en Guatemala en la Primera Decada del Siglo XXI [Superior Education in Guatemala during the first decade of XXI century].» Revista Innovacion Educativa 11, no 57 (2011): 69-80.

Torres, Milton Alfredo. «Los Politiqueros se Aduenan de la USAC [Political actors become owners of USAC].» La Hora, 19 de February de 2008: 8.

UNDP. Human Development Index Report "The Rise of the South". Research Report, New York: UNDP, 2013.

UNESCO. Situacion Educativa de America Latina $y$ el Caribe [Educational Situation of Latin America and the Caribbean]. Mexico City: National Secretariat for Public Education Mexico, 2012, 8996.

UNESCO/IESALC (Institute for Superior Education in Latin America]. Informe sobre la Educacion Superior en America Latina y el Caribe 2000-2005 [Report about superior edcuation in Latin America and the Caribbean 2000-2005]. Caracas: Editorial Metamorfosis C.A., 2006.

UNICEF. El Salto al Norte: Violencia e Impunidad del Fenomeno Migratorio en Guatemala [The jump north: violence and impunity of the migration phenomenon in Guatemala]. Guatemala City: UNICEF, 2010.

World Bank. Country Classifications. New York, 31 de 05 de 2013.

World Economic Forum. The Global Competitiveness Report. Geneveva: The Global Benchmarking Network, 2012. 
